\title{
Sturmian Theory \\ for a Class of Nonselfadjoint Differential Systems $\left(^{*}\right)\left({ }^{* *}\right)$.
}

\author{
M. S. KeEner (Stillwater, Ok.) - O. C. Travis (Oak Ridge, Tenn.)
}

Summary. - This paper investigates certain. Sturmian properties of the differential system $Y^{\prime \prime}+P(t) Y=0$, where $Y$ is a real $n$-dimensional vector and $P$ is a real continuous (possibily nonsymmetric) $n \times n$ matrix which is positive with respect to a solid cone $K$ in $R^{n}$. The presence or absence of focal points and conjugate points on a given interval is characterized in terms of integral inequalities on a Banach lattice. These inequalities are then used to give specific criteria for the presence or absence of a focal point or a conjugate point on a half-line $[\alpha, \infty)$. An example is given to demonstrate that in general the Sturmian properties of the above equation depend on whether $P$ is assumed symmetric or nonsymmetric.

\section{1. - Introduction.}

In this paper we investigate certain Sturmian properties of the second order differential system

$$
Y^{\prime \prime}+P(x) Y=0
$$

where $Y$ is a real $n$-dimensional vector and $P$ is a real continuous (possibly nonsymmetric) $n \times n$ matrix defined on an interval $I$. An extensive literature exists on the Sturmian properties of selfadjoint differential systems of the form (1.1). However, when $P$ is not assumed symmetric relatively few results exist and those that do are fragmentary in nature (see $[5],[6],[10],[14],[15])$. The primary purpose of the present study is to demonstrate that the theory of $\mu_{0}$-positive operators defined on a Banach space equipped with a cone can be employed to systematically develop the Sturmian properties of nonselfadjoint differential systems. The strength of this method is reflected in the fact that almost all of the results obtained are new even in the selfadjoint case.

Throughout this paper we will assume that $P$ satisfies the following positivity condition:

there exists a solid cone $K$ in $R^{n}$ with interior $K^{0}$ such that $P(t) v \in K^{0}$ for $t \in I$ whenever $v \in K, v \neq \mathbf{0}$.

(*) Entrata in Redazione il 15 novembre 1978.

(**) Operated by Union Carbide Corporation for the Department of Energy. 
This positivity condition is an abstraction of the concept of a matrix with positive entries. If $P(t)=\left(P_{i j}(t)\right)$ and $P_{i j}(t)>0$ for $t \in I$, then this condition can be satisfied by choosing the cone $K=\left\{\left(x_{1}, x_{2}, \ldots, x_{n}\right) \in R^{n} \mid x_{i} \geqslant 0\right\}$. AHyrad and L LZER ([1], [2]) have studied equation (1.1) where the coefficient $P$ is positive with respect to this particular cone. The results presented here for conjugate points parallel some of their results. However, the present results are by no means restricted to this case. Suppose, for example, that $P(t)=\left(P_{i j}(t)\right)$ and $(-1)^{i+j} P_{i j}(t)>0$ for $t \in I$. The positivity condition is satisfied by the cone

$$
K=\left\{\left(x_{1}, x_{2}, \ldots, x_{n}\right) \in R^{n} \mid(-1)^{i} x_{i} \geqslant 0\right\} .
$$

Another example is provided by the constant matrix

$$
P=\left(\begin{array}{rr}
3 & 0 \\
-1 & 1
\end{array}\right)
$$

This matrix satisfies the positivity condition with the choice of the cone $K=\{(x$, $\left.y) \in R^{2}|0 \leqslant x| y \mid, \leqslant x\right\}$.

In this study we will investigate properties of focal points and conjugate points relative to equation (1.1). If $\alpha \in I$, a point $\eta(\alpha) \in I, \eta(\alpha)>\alpha$, is called a focal point of $\alpha$ relative to equation (1.1) provided there is a nontrivial solution $Y$ of (1.1) satisfying $Y(\alpha)=Y^{\prime}(\eta(\alpha))=0$, and there is no nontrivial solution of (1.1) which satisfies $Y(\alpha)=Y^{\prime}(\gamma)=0$ for $\alpha<\gamma<\eta(\alpha)$. A point $\delta(\alpha) \in I, \delta(\alpha)>\alpha$, is called a conjugate point of $\alpha$ relative to equation (1.1) provided there is a nontrivial solution of (1.1) satisfying $Y(\alpha)=Y(\delta(\alpha))=0$, and there is no nontrivial solution of (1.1) which satisfies $Y(\alpha)=Y(\gamma)=0$ for $\alpha<\gamma<\delta(\alpha)$. If equation (1.1) does not posses such a focal (conjugate) point on an interval $(\alpha, \beta) \subset I$ the equation will be said to be disfocal (disconjugate) on $[\alpha, \beta]$.

The primary purpose of the present study is to investigatie the relationship between the existence of focal points and conjugate points relative to equation (1.1), and the existence of certain functions contained in the positive cone of a particular Banach lattice which satisfy certain integral inequalities. ScHмIтT and SuITH [20] have considered the existence of conjugate points in a manner similar to that presented here. However, by considering both the focal point and conjugate point problems, explicit criteria can be obtained, in terms of the coefficient $P$, for the presence or absence of focal points and conjugate points of equation (1.1). The methods presented here are inspired by the work of $Z$. NEHARI [17] on the disconjugacy properties of the scaler differential equation $y^{(n)}-(-1)^{n-k} p(t) y=0$. NEHARI demonstrated that criteria for the presence or absence of conjugate points for this class of equations may be formulated in terms of integral inequalities involving appropriate Green's functions. This observation forms the basis for the present results. However, our formulation will be in the abstract setting of a Banach lattice and will rely heavily on the abstract theory of $\mu_{0}$-positive operators. 
Section 2 contains some basic results concerning eigenvalues of operators defined on a Banach space $\mathcal{B}$ which are $\mu_{0}$-positive with respect to a cone $\mathcal{T}$. We then introduce the integral operators corresponding to the focal point eigenvalue problem

$$
Y^{\prime \prime}+\lambda P(t) Y=0, \quad Y(\alpha)=Y^{\prime}(\beta)=0,
$$

and the conjugate point eigenvalue problem

$$
\bar{Y}^{\prime \prime}+\lambda P(t) \bar{Y}=0, \quad \bar{Y}(\alpha)=\bar{Y}(\beta)=0 .
$$

An application of the theory of $\mu_{0}$-positive operators establishes the existence of a smallest positive eigenvalue for each of these eigenvalue problems.

Section 3 contains necessary and sufficient conditions for the absence of focal and conjugate points of equation (1.1) in terms of integral and differential inequalities on a Banach lattice. In Section 4 we obtain necessary and sufficient conditions for the existence of focal and conjugate points on a finite interval $(\alpha, \beta)$ in terms of integral and differential inequalities on a Banach lattice. These conditions are employed to establish the following result:

Proposition 4.6. - Equation (1.1) has a conjugate point $\delta(\alpha)$ on the interval $(\alpha, \infty)$ if and only if it has a focal point $\eta(\alpha)$ on this interval.

This proposition demonstrates that on a semi-infinite interval $(\alpha, \infty)$ it is unimportant whether we consider the presence of conjugate points or that of focal points. The importance of this observation, as presented in Section 5, is that explicit criteria for the presence or absence of focal points are easier to obtain than the corresponding criteria for conjugate points. For example, assume that

$$
P(t)=\left(p_{i j}(t)\right), \quad p_{i j}(t)>0
$$

for $1 \leqslant i, j \leqslant n$ on $[0, \infty)$, and set

$$
\begin{aligned}
& \|P(t)\|_{\infty}=\max _{1 \leqslant i \leqslant n} \sum_{j=1}^{n} P_{i j}(t) \\
& \|P(t)\|_{1}=\max _{1 \leqslant j \leqslant n} \sum_{i=1}^{n} p_{i j}(t) .
\end{aligned}
$$

Then sufficient conditions for equation (1.1) to be disfocal (and hence disconjugate) on the interval $[0, \infty)$ are that

$$
\int_{t}^{\infty} s\|P(s)\|_{\infty} d s \leqslant 1, \quad t \geqslant 0,
$$

or

$$
\int_{i}^{\infty} s\left\|_{i} P(s)\right\|_{1} d s \leqslant 1, \quad t \geqslant 0
$$


while necessary conditions for equation (1.1) to be disfocal on the interval $[0, \infty)$ are that

$$
i \int_{i}^{\infty}\|\boldsymbol{P}(s)\|_{\infty} d s \leqslant 1, \quad t \geqslant 0,
$$

or

$$
i \int_{t}^{\infty}\|P(s)\|_{1} d s \leqslant 1, \quad t \geqslant 0
$$

At this point we would like to remark that the Sturmian properties of nonselfadjoint differential systems of the form (1.1) are not necessarily the same as those of selfadjoint systems. For example, if $P(t)=\left(P_{i j}(t)\right)$ is an $n \times n$ symmetric matrix with $P_{i j} \geqslant 0,1 \leqslant i, j \leqslant n$, then it is known [4] that a sufficient condition for the existence of a conjugate point $\delta(\alpha)$ of $\alpha$ relative to equation (1.1) for every $\alpha$ in the interval $(\beta, \infty)$ is that

$$
\int_{\beta}^{\infty} P_{i j}(s) d s=+\infty
$$

for some pair $(i, j), 1 \leqslant i, j \leqslant n$. Recently AHMAD and Travis [3] have shown that this result is not valid without the assumption that $P$ is symmetric. They consider equation (1.1) on the interval $(1, \infty)$ with $P$ defined by

$$
P(t)=\left(\begin{array}{lr}
1 / 4 t^{2}, & 0 \\
1, & 1 / 4 t^{2}
\end{array}\right),
$$

and show that for $\alpha$ sufficiently large equation (1.1) is disconjugate on the interval $[\alpha, \infty)$ even though $\int_{1}^{\infty} P_{21}(s) d s=+\infty$. The reader should therefore bear in mind that while some Sturmian properties which are valid for selfadjoint systems are also valid for nonselfadjoint systems, in general this is not the case.

\section{2. $-\mu_{0}$-positive operators.}

Let $\mathfrak{B}$ denote a real Banach space. A closed subset $\mathfrak{S} \subset \mathcal{B}$ is called a cone provided it satisfies the following conditions:

i) $u \in \mathfrak{T}$ and $v \in \mathfrak{T}$ implies $u+v \in \mathfrak{T}$,

ii) $u \in \mathfrak{T}$ and $t \geqslant 0$ implies $t u \in \mathfrak{T}$,

iii) $u \in \mathfrak{T}$ and $-u \in \mathfrak{T}$ implies $u=0$. 
A cone $\mathfrak{T}$ is said to be solid provided its interior $\mathscr{T}_{0}$ is nonempty. Every cone $\mathfrak{T}$ on a Banach space $\mathfrak{B}$ generates a partial ordering on both the space $\mathscr{B}$ and the space of bounded linear operators mapping $\mathfrak{B}$ into itself. For a given cone $\mathfrak{S}$ of a Banach space $\mathfrak{B}$ and elements $u, v \in \mathfrak{B}$ we will say that $L u \leqslant v$ with respect to $\mathcal{T}$ provided $v-u \in \mathfrak{S}$. Moreover, if $\mathfrak{T}$ is solid, we will say $u<v$ with respect to $\mathfrak{T}$ provided $v-u \in \mathfrak{T}$. For bounded linar operators $M$ and $L$ mapping $\mathfrak{B}$ into itself, we shall say that $M \leqslant L$ with respect to $\mathfrak{T}$ provided $M u \leqslant L v$ for all $u \in \mathfrak{T}$; and, if $P$ is solid, we shall say that $M<L$ with respect to $\mathfrak{T}$ provided $M u \leqslant L u$ for all nonzero $u \in \mathfrak{T}^{0}$.

A bounded linear operator $M: \mathfrak{B} \rightarrow \mathfrak{B}$ is said to be $\mu_{0}$-positive (with respect to a cone $\mathfrak{T} \subset \mathfrak{B}$ ) provided there exists a nonzero element $\mu_{0} \in \mathfrak{S}$ such that for every nonzero element $u \in \mathfrak{T}$, there exists positive constants $k_{1}$ and $k_{2}$ and a positive integer $n$ such that

$$
k_{1} \mu_{0} \leqslant M^{n} u \leqslant k_{2} \mu_{0}
$$

with respect to the cone $\mathfrak{T}$.

The class of $\mu_{0}$-positive operators has been studied by M. A. KrasnosELsKII and L. A. LADyzensKiI. The interested reader is referred to [13] for an extensive development of their theory and applications. The following theorem is one of the fundamental properties of compact $\mu_{0}$-positive linear operators defined on a Banach space equipped with a solid cone $\mathfrak{T}$.

Proposition 2.1. - If $L$ is a compact $\mu_{0}$-positive linear operator, then $L$ has exactly one (normalized) eigenvector in $\mathfrak{T}$ and the corresponding eigenvalue is simple, positive, and larger than the absolute value of any other eigenvalue.

The following proposition is an extension of a result of C. C. TRAvIs [22, p. 365] concerning the comparison of eigenvalues of $\mu_{0}$-positive operators. The proof is similar to that in [22] and therefore will be omitted.

Proposition 2.2. - Let $L$ and $M$ be bounded linear operators and assume that at least one of the operators is $\mu_{0}$-positive. If $L \leqslant M$ and

$$
L u_{1} \geqslant \lambda_{1} u_{1} \quad\left(u_{1} \in \mathfrak{T}, \lambda_{1}>0\right)
$$

and

$$
L u_{2} \leqslant \lambda_{2} u_{2} \quad\left(u_{2} \in \mathfrak{T}, \lambda_{2}>0\right),
$$

then $\lambda_{1} \leqslant \lambda_{2}$, and if $\lambda_{1}=\lambda_{2}, u_{1}$ is a scaler multiple of $u_{2}$.

Consider now the differential operator

$$
D[f]=-f^{\prime \prime}
$$

subject either to the focal point boundary conditions

$$
f(\alpha)=f^{\prime}(\beta)=0
$$


or the conjugate point boundary conditions

$$
f(\alpha)=f(\beta)=0 .
$$

It is easily verified that the Green's functions for this differential operator subject to the boundary conditions (2.2) and (2.3) are given by

$$
G(t, s)= \begin{cases}t-\alpha, & \alpha \leqslant t \leqslant s \leqslant \beta \\ s-\alpha, & \alpha \leqslant s \leqslant t \leqslant \beta\end{cases}
$$

and

$$
g(i, s)= \begin{cases}\frac{(t-\alpha)(\beta-s)}{\beta-\alpha}, & \alpha \leqslant t \leqslant s \leqslant \beta \\ \frac{(s-\alpha)(\beta-t)}{\beta-\alpha}, & \alpha \leqslant s \leqslant t \leqslant \beta\end{cases}
$$

respectively.

Rather than studying the eigenvalue problems (1.2) and (1.3) directly, consider the related integral operators

$$
M[u]=\int_{\alpha}^{\beta} G(\cdot, s) P(s) u(s) d s
$$

and

$$
L[u]=\int_{\alpha}^{\beta} g(\cdot, s) \cdot P(s) u(s) d s
$$

defined on the Banach space

$$
\Re=\left\{u \in O\left([\alpha, \beta] ; \mathbb{R}^{n}\right) \mid u(\alpha)=0\right\}
$$

equipped with the norm $\|u\|=\sup _{\alpha \leqslant t \leqslant \beta}\|u(t)\|_{\boldsymbol{R}^{n}}$

Let $K$ be the solid cone in $R^{n}$ such that $P(t) v \in K^{0}$ for $t \in(\alpha, \beta)$ and all nonzero $v \in K$, and consider the solid cone $\mathfrak{T}(\beta) \subset \mathfrak{B}$ defined by

$$
\mathfrak{T}(\beta)=\{u \in \mathfrak{B} \mid u(t) \in \mathbb{K} \text { for } t \in[\alpha, \beta]\}
$$

with interior $\mathfrak{T}^{0}(\beta)=\left\{u \in \mathfrak{B} \mid u(t) \in K^{0}\right.$ for $\left.t \in(\alpha, \beta)\right\}$. We wish to show that the operators (2.6) and (2.7) are compact $\mu_{0}$-positive operators with respect to the cone $\mathfrak{T}(\beta)$. The following lemma will be needed. For $S \subset R^{n}$ the convex hull generated by $S$ is denoted by $C(S)$.

Lwirma 2.3. - Let $K$ denote a closed solid cone in $R^{n}$. Suppose $f:[\alpha, \beta] \rightarrow K$ is continuous and $f(t) \in \mathbb{K}^{0}$ for some $t \in[\alpha, \beta]$. Then $\int_{\alpha}^{\beta} f(s) d s \in K^{0}$. 
Proof. - Since $f$ is continuous and $f(t) \in K^{0}$, it follows that there exists a closed interval $\left[t_{1}, t_{2}\right] \subset[\alpha, \beta]$ for which $t \in\left[t_{1}, t_{2}\right]$ and $f(s) \in K_{0}$ for each $s \in\left[t_{1}, t_{2}\right]$. We shall first show that $1 /\left(t_{2}-t_{1}\right) \int_{t_{1}}^{t_{2}} f(s) d s$ (and hence $\int_{t_{2}}^{t_{2}} f(s) d s$ ) lies in $K^{0}$ : Let $I$ denote a partition of $\left[t_{1}, t_{2}\right]$ and consider a corresponding Riemann sum for this integral. Then

$$
\frac{1}{t_{2}-t_{1}} \sum_{i=1}^{i b} f\left(s_{i}\right) \Delta s_{i}=\sum_{i=1}^{n} f\left(s_{i}\right) \frac{\Delta s_{i}}{t_{2}-t_{1}} \in C\left(\left\{f\left(s_{i}\right)\right\}_{i=1}^{n}\right) \subset C\left(f\left(\left[t_{1}, t_{2}\right]\right)\right) \subset K^{0}
$$

Since the convex hull generated by a compact set in $R^{n}$ is itself compact, we have

$$
\frac{1}{t_{2}-t_{1}} \int_{t_{1}}^{t_{2}} f(s) d s \in K^{0} \text {. }
$$

Setting $\left.I=[\alpha, \beta] \backslash t_{1}, t_{2}\right]$ and applying the same analysis to the integral $\int_{I} f(s) d s$, it follows that $\int_{I} f(s) d s \in K$ since $K$ is closed. By the cone properties $\int_{\alpha}^{\beta} f(s) d s=$ $=\int_{I} f(s) d s+\int_{i_{1}}^{t_{0}} f(s) d s \in K_{\mathbf{I}}$, and the proof of the lemma is complete.

In view of the positivity condition on $K$ and the previous lemma, it is apparent that both the operators (2.6) and (2.7) map the cone $\mathcal{S}(\beta)$ into its interior $P^{0}(\beta)$. This observation shows that both operators are $\mu_{0}$-positive. Indeed, considering the operator (2.6), we choose $r_{0} \in K_{0}$ and set

$$
\mu_{0}(t)=\left(\int_{\alpha}^{\beta} G(t, s) d s\right) r_{0} \in \mathfrak{T}^{0}(\beta) .
$$

Let $u \in \mathfrak{T}(\beta)$ and observe that $M[u] \in \mathfrak{T}^{0}(\beta)$. Then for sufficiently small $k_{1}>0, M[u]-$ $-k_{1} u_{0} \in \mathfrak{T}(\beta)$, i.e., $k_{1} \mu_{0} \leqslant M[u]$. On the other hand, for sufficiently large $k_{2}>0$, $\mu_{0}-\left(1 / k_{2}\right) M[u] \in \mathfrak{T}(\beta)$, i.e., $N[u] \leqslant k_{2} \mu_{0}$. Hence $k_{1} \mu_{0} \leqslant M[u] \leqslant k_{2} \mu_{0}$ with respect to the cone $\mathfrak{T}(\beta)$. A similar proof may be given for the operator (2.7). The compactness of these operators may be established in the standard manner using Ascoli's Theorem. These remarks provide a proof of the following proposition.

Proposition 2.4. - The operators (2.6) and (2.7) are compact and $\mu_{0}$-positive with respect to the cone $\mathfrak{T}(\beta)$.

We are now able to state an existence theorem concerning the eigenvalue problems (1.2) and (1.3).

Proposition 2.5. - The focal point eigenvalue problem

$$
Y^{\prime \prime}+\lambda P(t) Y=0, \quad Y(\alpha)=Y^{\prime}(\beta)=0,
$$


and the conjugate point eigenvalue problem

$$
\Psi^{\prime \prime}+\lambda P(t) Y=0, \quad Y(\alpha)=Y(\beta)=0,
$$

each have a real eigenvalue $\lambda_{0}(\beta)$ which is simple, positive, and smaller than the absolute value of any other eigenvalue. The normalized eigenvectors associated with each of these eigenvalues are contained in the cone $\mathfrak{T}(\beta)$ and are the only eigenvalues with this property. Moreover, the smallest positive eigenvalue $\lambda_{0}(\beta)$ is a continuous, monotone decreasing function of the right endpoint $\beta$ of the interval $[\alpha, \beta)$ with the property that $\lim _{\beta \rightarrow \alpha+} \lambda_{0}(\beta)=+\infty$.

Proof. - The existence of a unique normalized eigenvector in the cone $\mathfrak{T}(\beta)$ and a corresponding eigenvector which is simple, positive, and smaller than the absolute value of any other eigenvalue follows from Propositions 2.1 and 2.4. The continuity and monotonicity properties of the smallest positive eigenvalue $\lambda_{0}(\beta)$ may be established as in [12] and [20].

\section{3. - Focal points and conjugate points.}

In this section we shall establish necessary and sufficient conditions for the absence of focal and conjugate points of equation (1.1). The first proposition establishes the fact that if equation (1.1) has a focal point or a conjugate point, then the extremal solution of (1.1) corresponding to either of these points lies in the cone $\mathcal{T}(\beta)$.

PRoposition 3.1. - If equation (1.1) has a focal point $\eta(\alpha)$, the extremal solution of (1.1) corresponding to the focal point is in the cone $\mathfrak{I}(\beta)$. Moreover $u(t) \in K^{0}$ for $t \in(\alpha$, $\eta(\alpha)]$. A similar result holds for the extremal solution corresponding to the conjugate point.

Proof. - We shall only give a proof for the focal point problem since the proof is similar for the conjugate point problem. If $\beta=\eta(\alpha)$ is the focal point corresponding to $\alpha$, then $\lambda=1$ is an eigenvalue for the focal point eigenvalue problem (2.8), and hence the smallest positive eigenvalue $\lambda_{0}(\beta) \leqslant 1$. If $\lambda_{0}(\beta)<1$, the monotonicity property in Proposition 2.5 implies the existence of a point $\beta_{1} \in(\alpha, \beta)$ for which $\lambda_{0}\left(\beta_{1}\right)=1$. However, this would imply that there is a nontrivial solution of (1.1) for which $Y(\alpha)=$ $=\bar{Y}^{\prime}\left(\beta_{1}\right)=0$. Since $\beta_{1} \in(\alpha, \beta)$, such a solution contradicts the definition of a focal point. Therefore $\lambda_{0}(\beta)=1$, and by Proposition 2.5 the corresponding eigenvector lies in the cone $\mathfrak{T}(\beta)$. This completes the proof of the proposition.

The next two propositions provide necessary and sufficient conditions for disfocality and disconjugacy on an interval in terms of inequalities involving the smallest positive eigenvalue $\lambda_{0}(\beta)$. These inequalities will be useful in establishing the integral inequalities on the Banach lattice.

Proposition 3.2. - A necessary and sufficient condition for equation (1.1) to be disfocal on the interval $[\alpha, \beta), \alpha<\beta \leqslant+\infty$, is that the smallest positive eigenvalue 
$\lambda_{0}(\bar{\beta})$ of the eigenvalue problem $(2.8)$ on the interval $[\alpha, \bar{\beta}]$ satisfies $1 \leqslant \lambda_{0}(\bar{\beta})$ for every $\bar{\beta}$ such that $\alpha<\bar{\beta}<\beta$.

Proof. - If equation (1.1) has a focal point $\beta_{1}=\eta(\alpha)$ in the interval $[\alpha, \beta)$, then the smallest positive eigenvalue of $(2.8)$ for the interval $\left[\alpha, \beta_{1}\right]$ is $\lambda_{0}\left(\beta_{1}\right)=1$ as seen in the proof of Proposition 3.1. Since $\lambda_{0}(\beta)$ is monotone decreasing as a function of the endpoint $\beta$ of the interval $[\alpha, \beta]$, it follows that $\lambda_{0}(\bar{\beta})<1$ for $\beta<\bar{\beta}$.

For the converse, assume that equation (1.1) is disfocal on the interval $[\alpha, \beta)$ and that there exists a point $\bar{\beta}$ such that $\alpha<\bar{\beta}<\beta$ and $\lambda_{0}(\bar{\beta})<1$. Using the continuity and monotonicity properties of $\lambda_{0}$ described in Proposition 2.5 , there exists a point $\beta_{1} \in(\alpha, \bar{\beta})$ for which $\lambda_{0}\left(\beta_{1}\right)=1$. This fact contradicts the supposition that equation (1.1) is disfocal on $[\alpha, \beta)$. This completes the proof of the proposition.

Using the smallest positive eigenvalue corresponding to the conjugate point eigenvalue problem (2.9) rather than (2.8), a similar procedure leads to the following characterization of disconjugacy.

Proposition 3.3. - A necessary and sufficient condition for equation (1.1) to be disconjugate on the interval $[\alpha, \beta), \alpha<\beta \leqslant+\infty$, is that the smallest positive eigenvalue $\lambda_{0}(\bar{\beta})$ of the eigenvalue problem $(2.9)$ on the interval $[\alpha, \bar{\beta}]$ satisfies $1 \leqslant \lambda_{0}(\bar{\beta})$ for every $\bar{\beta} \in(\alpha, \beta)$.

The following two results are fundamental to this study. They provide necessary and sufficient conditions in terms of integral inequalities on the Banach lattice $\mathscr{B}$ for the absence of focal points, or conjugate points, on a given finite interval.

Propostrion 3.4. - A necessary and sufficient condition for equation (1.1) to be disfocal on the finite interval $[\alpha, \beta)$ is that there exists a nontrivial function $v$ contained in the cone $\mathfrak{S}(\beta)$ such that

$$
\int_{\alpha}^{\beta} G(\cdot, s) P(s) v(s) d s \leqslant v
$$

with respect to the cone $\mathfrak{T}(\beta)$.

Proposition 3.5. - A necessary and sufficient condition for equation (1.1) to be disconjugate on the finite interval $[\alpha, \beta)$ is that there exists a nontrivial function v contained in the cone $\mathfrak{T}(\beta)$ such that

$$
\int_{\alpha}^{\beta} g(\cdot, s) P(s) v(s) d s \leqslant v
$$

with respect to the cone $\mathfrak{S}(\beta)$.

The proof of Proposition 3.5 is similar to the proof of Proposition 3.4. To prove Proposition 3.4, assume first that equation (1.1) is disfocal on the interval $[\alpha, \beta)$. By Proposition 3.2 the smallest positive eigenvalue $\lambda_{0}(\beta)$ of the eigenvalue problem 
(2.8) satisfies $\lambda_{0}(\beta) \geqslant 1$. Let $v$ denote the normalized eigenfunction contained in the cone $\mathfrak{T}(\beta)$ corresponding to $\lambda_{0}(\beta)$. Then

$$
\int_{\alpha}^{\beta} G(\cdot, s) P(s) v(s) d s=\lambda_{0}^{-1}(\beta) v \leqslant v
$$

with respect to the cone $\mathfrak{T}(\beta)$.

To prove the converse, suppose that there exists a nontrivial function $v$ contained in the cone $\mathfrak{T}(\beta)$ such that

$$
M[v]=\int_{\alpha}^{\beta} G(\cdot, s) P(s) v(s) d s \leqslant v
$$

with respect to the cone $\int(\beta)$. Letting $\lambda_{0}(\beta)$ denote the smallest positive eigenvalue for the eigenvalue problem (2.8) and $w$ its corresponding normalized eigenfunction, we have $M[w]=\lambda_{0}^{-1}(\beta) w$. Since $M$ is a compact $\mu_{0}$-positive operator, it follows from Proposition 2.2 that $I \leqslant \lambda_{0}(\beta)$. Proposition 3.2 then implies that equation (1.1) is disfocal on $[\alpha, \beta)$, and the proof of the proposition is complete.

The next two results provide necessary and sufficient conditions in terms of differential inequalities on the Banach lattice for the absence of focal or conjugate points on a given finite interval. Again, we present only the proof for the focal point problem since the conjugate point problem may be handled in a similar fashion.

CoRollary 3.6. - A necessary and sufficient condition for equation (1.1) to be disfocal on the finite interval $[\alpha, \beta)$ is that there exists a nontrivial function $v$ contained in the cone $\mathfrak{T}(\beta)$ such that $v^{\prime}(\beta) \in K$ and

$$
v^{\prime \prime}+P v \leqslant 0
$$

with respect to the cone $\mathfrak{T}(\beta)$.

Coroldary 3.7. - A necessary and sufficient condition for equation (1.1) to be disconjugate on the finite interval $[\alpha, \beta)$ is that there exists a nontrivial function $v$ contained in the cone $\mathfrak{T}(\beta)$ such that

$$
v^{n}+P v \leqslant 0
$$

with respect to ine cone $\mathcal{T}(\beta)$.

Proof. - To verify Corollary 3.6, first assume that equation (1.1) is disfocal on the interval $[\alpha, \beta)$. Then the normalized eigenvector $v$ of the eigenvalue problem (2.8) satisfies $v^{\prime}(\beta)=0 \in \mathbb{K}, v \in \mathfrak{T}(\beta)$, and

$$
v^{\prime \prime}+\lambda_{0}(\beta) P v=0 .
$$


Since $\lambda_{0}(\beta) \geqslant 1$, we have

$$
v^{\prime \prime}+P v \leqslant v^{\prime \prime}+\lambda_{0}(\beta) P v=0
$$

with respect to the cone $\mathscr{T}(\beta)$.

For the sufficiency of the condition, assume that there exists a nontrivial function $v$ contained in the cone $\mathfrak{T}(\beta)$ such that $v^{\prime}(\beta) \in K$ and (3.3) is satisfied, i.e., for $s \in[\alpha, \beta]$

$$
-v^{\prime \prime}(s)-P(s) v(s) \in K
$$

Multiplying (3.5) by $G(t, s)$ and integrating with respect to $s$ over $[\alpha, \beta]$ yields by Lemma 2.3

$$
v(t)-(t-\alpha) v^{\prime}(\beta)-\int_{\alpha}^{\beta} G(t, s) P(s) v(s) d s \in K .
$$

Since $v^{\prime}(\beta) \in K$, we have

$$
v(t) \geqslant \int_{\alpha}^{\beta} G(t, s) P(s) v(s) d s, \quad t \in[\alpha, \beta] .
$$

Applying Proposition 3.4, the proof of the corollary is complete.

It should be pointed out that Corollary 3.7 is a generalization of known results for sealer equations. S. AHMAD has communicated to the authors a result similar to Corollary 3.7 for the particular case $P(t)=\left(P_{i j}(t)\right), p_{i j}(t)>0,1 \leqslant i, j \leqslant n$, on $[\alpha, \beta]$. Also for the focal point problem more may be said about the function $v$ in Corollary 3.6. Namely, in the case that equation (1.1) is disfocal on $[\alpha, \beta]$, not only is $v^{\prime}(\beta) \in K$ but also $v^{\prime}(t) \in K_{0}$ for all $t \in[\alpha, \beta)$. To see this, let $v$ be as in Corollary 3.6 and satisfy the inequality (3.5). Integrating (3.5) over the interval $[t, \beta]$ yields by Lemma 2.3

$$
v^{\prime}(t)-v^{\prime}(\beta)-\int_{i}^{\beta} P(s) v(s) d s \in K
$$

from which it follows that $v^{\prime}(t) \in K^{0}$ for $t \in[\alpha, \beta]$.

We conclude this section by noting that disfocality on an interval implies disconjugacy on this interval. Indeed the proof consists of merely comparing Corollaries 3.6 and 3.7.

COROLLARY 3.8. - If equation (1.1) is disfosal on the interval $[\alpha, \beta]$, then it is disconjugate on the interval $[\alpha, \beta]$.

\section{4. - More on focal and conjugate points.}

It is also possible to obtain necessary and sufficient conditions for the existence of focal points and conjugate points on a finite interval $(\alpha, \beta]$ in terms of differential and integral inequalities on the Banach lattice $\mathcal{B}$. We begin by considering integral inequalities for the existence of focal and conjugate points.

17 - Annali ai Matematica 
Proposimion 4.1. - A necessary and sufficient condition for the interval $(\alpha, \beta]$ to contain a focal point of $\alpha$ relative to equation (1.1) is that there exists a nontrivial function $v$ contained in the cone $\mathfrak{T}(\beta)$ such that

$$
\int_{\alpha}^{\beta} G(\cdot, s) P(s) v(s) d s \geqslant v
$$

with respect to the cone $\mathfrak{S}(\beta)$.

Proposition 4.2. - A necessary and sufficient condition for the interval $(\alpha, \beta]$ to contain a conjugate point of $\alpha$ relative to equation (1.1) is that there exists a nontrivial function $v$ contained in the cone $\mathfrak{S}(\beta)$ such that

$$
\int_{a}^{\beta} g(\cdot, s) P(s) v(s) d s \geqslant v
$$

with respect to the cone $\mathcal{S}(\beta)$.

Proof. - The proof of Proposition 4.2 is similar to that of Proposition 4.1 and therefore is omitted. The proof of Proposition 4.1 proceeds as follows. If equation (1.1) has a focal point $\eta(\alpha)=\beta_{1} \in(\alpha, \beta]$, then the smallest positive eigenvalue $\lambda_{0}\left(\beta_{1}\right)$ of the eigenvalue problem (2.8) on the interval $\left[\alpha, \beta_{1}\right]$ satisfies $\lambda_{0}\left(\beta_{1}\right)=1$. Since $\beta_{1} \leqslant \beta$, we have by the monoticity of $\lambda_{0}$

$$
0<\lambda_{0}(\beta) \leqslant \lambda_{0}\left(\beta_{1}\right)=1
$$

Let $v$ be the normalized eigenfunction contained in the cone $\mathscr{T}(\beta)$ corresponding to $\lambda_{0}(\beta)$. Then

$$
\int_{\alpha}^{\beta} G(\cdot, s) P(s) v(s) d s=\lambda_{0}^{-1}(\beta) v \geqslant v
$$

with respect to the cone $\mathscr{S}(\beta)$.

Conversely, suppose that there exists a nontrivial function $v$ contained in the cone $\mathscr{T}(\beta)$ such that

$$
M[v]=\int_{\alpha}^{\beta} G(\cdot, s) P(s) v(s) d s \geqslant v
$$

with respect to the cone $\mathfrak{T}(\beta)$. Letting $\lambda_{0}(\beta)$ be the smallest positive eigenvalue of $(2.8)$ and $w$ the corresponding normalized eigenfunction, we have $M[w]=\lambda_{0}^{-1}(\beta) w$. If follows from (4.4) and Proposition 2.2 that $\lambda_{0}(\beta) \leqslant 1$. Proposition 3.2 then implies equation (1.1) has a focal point $\eta(\alpha)$ in the interval $(\alpha, \beta]$, and the proof of the proposition is complete. 
The following corollaries may be proved in a manner similar to that used in Section 3 for Corollaries (3.6) and (3.7). For this reason their proofs are omitted.

COROLLARy 4.3. - A necessary and sufficient conditions for the interval $(\alpha, \beta]$ to contain a focal point of $\alpha$ relative to equation (1.1) is that there exists a nontrivial function $v$ contained in the cone $\mathcal{S}(\beta)$ such that $v^{\prime}(\beta) \leqslant 0$ and

$$
v^{\prime \prime}+P v \geqslant 0
$$

with respect to the cone $\mathfrak{S}(\beta)$.

COROLLARY 4.4. - A necessary and sufficient condition for the interval $(\alpha, \beta]$ to contain a conjugate point of $\alpha$ relative to equation (1.1) is that there exists a nontrivial function $v$ contained in the cone $T(\beta)$ such that $v(\beta)=0$ and

$$
v^{n}+P v \geqslant 0
$$

with respect to the cone $\mathfrak{T}(\beta)$.

We now present a result connecting the presence of a conjugate point for equation (1.1) on the interval $(\alpha, \infty)$ with the presence of a focal point on this same interval. Recalling Corollary 3.8 it is clear that if $\delta(\alpha)$ exists, then $\eta(\alpha)$ exists and $\eta(\alpha) \leqslant \delta(\alpha)$. Furthermore, it is clear that the existence of $\eta(\alpha)$ on a finite interval does not necessarily imply the existence of $\delta(\alpha)$ on this same interval. However, as the following proposition demonstrates, on a half-line $(\alpha, \infty)$ the existence of either $\eta(\alpha)$ or $\delta(\alpha)$ implies the existence of the other.

Proposition 4.5. - Equation (1.1) has a conjugate point $\delta(\alpha)$ on the interval $(\alpha, \infty)$ if and only if it has a focal point $\eta(\alpha)$ on this interval.

Proof. - From the remarks preceding the statement of the proposition, it is clear we need only show that the existence of the focal point $\eta(\alpha)$ on $(\alpha, \infty)$ implies the existence of $\delta(\alpha)$ on $(\alpha, \infty)$. Suppose to the contrary that $\alpha$ has no conjugate point on $(\alpha, \infty)$ relative to equation (1.1), i.e., for each $\gamma \in(\alpha, \infty)$ equation (1.1) is disconjugate on $[\alpha, \gamma)$. Let $\beta>\alpha$ and we shall show that under these assumptions equation (1.1) does not have a focal point on $[\alpha, \beta)$. Since $\beta$ is arbitrary, the proof will be complete.

For each $\gamma>\beta$, there exists an eigenvector $v_{\gamma} \in \mathfrak{T}(\gamma) \subset \mathfrak{J}(\beta)$ of the eigenvalue problem

$$
v_{\gamma}^{\prime \prime}+\lambda_{0}(\gamma) P v_{\gamma}=0 \quad v_{\gamma}(\alpha)=v_{\gamma}(\gamma)=0 .
$$

Since equation (1.1) is assumed disconjugate on $[\alpha, \gamma)$, we have by Proposition 3.1 
that $\lambda_{0}(\gamma) \geqslant 1$. It follows that $v_{y}$ satisfies the integral inequality

$$
\int_{\alpha}^{\nu} g_{\gamma}(\cdot, s) P(s) v_{\gamma}(s) d s \leqslant v_{\nu},
$$

with respect to the cone $\int(r)$. The symbol $g_{y}$ appearing in inequality (4.8) refers to the Green's function corresponding to the interval $[\alpha, \gamma]$ (recall $(2.5)$ ). We note in passing that $G(x, s)$ (see (2.4)) has no dependence on the right-hand endpoint of the interval, and so no distinction is necessary in this case. Returning to $g_{\gamma}$, a calculation shows that

$$
g_{\beta}(x, s)=g_{\gamma}(x, s)-\frac{(x-\alpha)(s-\alpha)(\gamma-\beta)}{(\gamma-\alpha)(\beta-\alpha)}
$$

A straight forward calculation together with (4.8) and (4.9) yields for $x \in[\alpha, \beta]$,

$$
\begin{gathered}
\int_{\alpha}^{\beta} G(x, s) P(s) v_{\gamma}(s) d s=\int_{\alpha}^{\beta} g_{\beta}(x, s) P(s) v_{\gamma}(s) d s+\int_{\alpha}^{\beta} \frac{(x-\alpha)(s-\alpha)}{\beta-\alpha} P(s) v_{\gamma}(s) d s \\
\quad=\int_{\alpha}^{\beta} g_{\gamma}(x, s) P(s) v_{\gamma}(s) d s+\frac{x-\alpha}{\gamma-\alpha} \int_{\alpha}^{\beta}(s-\alpha) P(s) v_{\gamma}(s) d s \\
\leqslant v_{\gamma}(s)+\frac{x-\alpha}{\gamma-\alpha} \int_{\alpha}^{\beta}(s-\alpha) P(s) v_{\gamma}(s) d s
\end{gathered}
$$

with respect to the cone $\mathbb{K}$. Since $\lambda_{0}(\gamma)$ is a monotone decreasing function which is bounded below, we may assume that

$$
\lim _{\gamma \rightarrow \infty} \lambda_{0}(\gamma)=\lambda_{0}(\infty) \geqslant 1
$$

Multiplying equation (4.7) by a suitable positive constant, we may assume without loss of generality, that $\lim _{\gamma \rightarrow \infty} v_{\gamma}^{\prime}(\alpha)=r$, where $\|v\|=1$. If $v(t)$ denotes the solution of the initial value problem

$$
v^{\prime \prime}+\lambda_{0}(\infty) P v=0, \quad v(\alpha)=0, \quad v^{\prime}(\alpha)=r,
$$

then by standard results concerning the continuity of solutions of differential equations with respect to initial conditions and parameters, it follows that $\lim _{\gamma \rightarrow \infty} v_{\gamma}(t)=v(t)$ uniformly on compact subintervals of $[\alpha, \infty)$. We also obtain that $v \in \mathfrak{T}(\beta)$ since $v_{\gamma}$ is contained in the closed cone $T(\beta)$. From the inequality (4.10), it is evident that

$$
\int_{\alpha}^{\beta} G(\cdot, s) P(s) v(s) d s \leqslant v,
$$

with respect to the cone $\mathfrak{T}(\beta)$. Applying Proposition 3.4, equation (1.1) is disfocal on the interval $[\alpha, \beta)$ and the proof of the proposition is complete. 


\section{5. - Criteria for the existence of a focal point.}

In this section we consider the relationship between the focal point problem for the equation (1.1) and the existence of a nontrivial function satisfying certain integral inequalities with respect to a narrower cone than $\mathfrak{S}(\beta)$. This will enable us to determine specific conditions on the coefficient $P$ in equation (1.1) for the presence or absence of a focal point on a given interval.

Recall from the discussion following the proof of Corollary 3.6, the function $v$ satisfying the differential inequality $v^{\prime \prime}+P v \leqslant 0$ with respect to the cone $\mathfrak{T}(\beta)$ has the property that both $v$ and $v^{\prime}$ lie in the cone $\mathfrak{T}(\beta)$. In view of these remarks we reformulate the focal point problem along the lines of Section 2 but with a different cboice of cones. Consider the differential operator $D u=-u^{\prime \prime}$ subject to the focal point boundary conditions $u(\alpha)=u^{\prime}(\beta)=0$ and, using the associated Green's function (2.4), define the integral operator

$$
M[u]=\int_{\alpha}^{\beta} G(\cdot, s) P(s) u(s) d s
$$

on the same Banach space $\mathcal{B}=\left\{u \in C\left([\alpha, \beta] ; R^{n}(\mid u(\alpha)=0\}\right.\right.$. But this time we equip $\mathcal{B}$ with the narrower cone $Q(\beta)$ of functions in $\mathfrak{B}$ for which $u^{(i)}(t) \geqslant 0$ with respect to the cone $K$ on $[\alpha, \beta]$ for $i=0,1$. Note that the only difference between this formulation and the sone in Section 2 is the choice of the cones. Following the same analysis as in Section 2, $M$ is a compact $\mu_{0}$-positive operator with respect to the cone $Q(\beta)$, and the results of Sections 3 and 4 on focal points also hold with respect to this narrower cone $Q(\beta)$. For reference we state the analogues of Propositions 3.4 and 4.1.

Proposition 5.1. - A necessary and sufficient condition for equation (1.1) to be disfocal on the finite interval $[\alpha, \beta)$ is that there exists a nontrivial function $v$ contained in the cone $Q(\beta)$ such that

$$
\int_{\alpha}^{\beta} G(\cdot, s) P(s) v(s) d s \leqslant v
$$

with respect to the cone $Q(\beta)$.

Proposition 5.2. - A necessary and sufficient condition for the interval $(\alpha, \beta]$ to contain a focal point of relative to equation (1.1) is that there exists a nontrivial function $v$ contained in the cone $Q(\beta)$ such that

$$
\int_{\alpha}^{\beta} G(\cdot, s) P(s) v(s) d s \geqslant v
$$

with respect to the cone $Q(\beta)$.

The following simpler characterization of disfocality can now be given. 
Proposimion 5.3. - A necessary and sufficient condition for equation (1.1) to be disfocal on the finite interval $[\alpha, \beta]$ is that there exists a nontrivial function $v$ contained in the cone $Q(\beta)$ such that for $t \in[\alpha, \beta]$

$$
\int_{i}^{\beta} P(s) v(s) d s \leqslant v^{\prime}(t)
$$

with respect to the cone $\mathbb{K}$.

Proof. - Assuming that equation (1.1) is disfocal on $[\alpha, \beta)$, the result follows immediately from Proposition 5.1. To prove the converse, suppose that such a function $\gamma \in Q(\beta)$ exists. Integrating (5.3) over the interval $[\alpha, t]$ and recalling that $v(\alpha)=0$, we have inequality (5.1). The proof of the proposition is then complete.

Due to Proposition 5.3 we may obtain explicit sufficient conditions on the coefficient $P$ for equation (1.1) to be disfocal on $[\alpha, \beta)$. For example, consider $v(t)=$ $=(t-\alpha) r$, where $0 \neq r=\left(r_{i}\right) \in K$. Then $v \in Q(\beta)$ and condition 5.3 becomes

$$
\int_{i}^{\beta}(s-\alpha) P(s) r d s \leqslant r
$$

with respect to the cone $K$ for $t \subset[\alpha, \beta]$. Furthermore if

$$
\int_{i}^{\alpha}(s-\alpha) P(s) r d s \leqslant r, \quad t \in[\alpha, \infty)
$$

with respect to the sone $K$ we may conclude that equation is disfocal (and hence disconjugate) on $[\alpha, \beta)$.

Let us consider $P(t)=\left(p_{i j}(i)\right)$, where $(-1)^{i+j} p_{i j}(t)>0,1 \leqslant i, j \leqslant n$, on $[\alpha, \infty)$. We noted in Section $I$ that $P$ was positive with respect to the cone $K=\left\{\left(x_{i}\right) \in\right.$ $\left.\in R^{n} \mid(-1)^{i} x_{i} \geqslant 0\right\}$. If we choose $r=\left((-1)^{i}\right)$, then condition (5.5) becomes

$$
\int_{i}^{\infty}(s-\alpha) \sum_{j=1}^{n}\left|p_{i j}(s)\right| d s \leqslant 1, \quad i=1,2, \ldots, n,
$$

for $i \in[\alpha, \infty)$.

If $P(t)=\left(p_{i j}(t)\right)$, where $p_{i j}(t)>0,1 \leqslant i, j \leqslant n$, on $[0, \infty)$, we may set $\|P(t)\|_{\infty}=$ $=\max _{1 \leqslant i \leqslant n} \sum_{j=1}^{n} p_{i j}(t)$ and select $K$ to be the traditional cone in $R^{n}$. Then condition (5.5) may be replaced by (1.4) namely,

$$
\int_{t}^{\infty} s\|P(s)\|_{\infty} d s, \quad t \geqslant 0 .
$$

It is easy to see that similar sufficient conditions may be given for coefficients $P$ which are positive with respect to a given cone $K$. 
Let us now consider the eigenvalue problem

$$
Z^{\prime \prime}+\mu P^{*}(t) Z=0, \quad Z(\alpha)=Z^{\prime}(\beta)=0,
$$

where $P^{*}$ is the transpose of the coefficient matrix $P$ in (2.8). Since $P^{*}$ is positive with respect to the solid cone

$$
K^{*}=\left\{v \in E^{n}:(v, \mu) \geqslant 0, \mu \in K\right\},
$$

we may conclude that there exists a smallest positive eigenvalue $\mu_{0}$ for the focal eigenvalue problem (5.6) and that the corresponding normalized eigenfunction $w$ is in the cone

$$
P^{*}(\beta)=\left\{\mu \in B: \mu(t) \in K^{*} \text { for } t \in[\alpha, \beta]\right\}
$$

If we let $\lambda_{0}$ and $v$ denote the smallest positive eigenvalue and normalized eigenfunction, respectively, for the problem (2.8), then for $t \in[\alpha, \beta]$,

$$
w^{\prime \prime}(t)+\mu_{0} P(t) w(t)=0, \quad w(\alpha)=w^{\prime}(\beta)=0
$$

and

$$
v^{\prime \prime}(t)+\lambda_{0} P(t) v(t)=0, \quad v(\alpha)=v^{\prime}(\beta)=0 .
$$

If follows from (5.7) and (5.8) that

$$
0=\left(\lambda_{0}-\mu_{0}\right) \int_{\alpha}^{\beta}(w(t), P(t) v(t)) d t .
$$

Since $v(t) \in K^{0}$ for $\alpha<t \leqslant \beta$, it follows that $P(t) v(t) \in K^{0}$ for $\alpha<t \leqslant \beta$. Hence

$$
\int_{\alpha}^{\beta}(w(t), P(t) v(t)) d t>0
$$

and we may conclude that $\lambda_{0}=\mu_{0}$. Consequently, the focal point $\eta^{*}(\alpha)$ of

$$
Z^{\prime \prime}+P^{*}(t) Z=0
$$

coincides with the focal point $\eta(\alpha)$ of (1.1) if they exist. We summarize these remarks these remarks in the following proposition.

Propositror 5.4. - Equation (1.1) and (5.9) are either both disfocal on $[\alpha, \beta)$ or the focal points of $\alpha$ relative to equation (1.1) and (5.9) coincide. A similar statement holds for conjugate points. 
In view of this proposition we may apply condition $(5.5)$ to $P^{*}$. Setting

$$
\|P(t)\|_{1}=\|P *(t)\|_{\infty}
$$

a sufficient condition for equation (1.1) to be disfocal on $[0, \infty)$ is $(1.5)$, namely

$$
\int_{i}^{\infty} s\|P(s)\|_{1} d s \leqslant 1, \quad t \geqslant 0 .
$$

The following corollary follows trivally form Proposition 4.1.

COROLLARY 5.5. - A sufficient condition for the interval $(\alpha, \beta]$ to contain a focal point of $\alpha$ relative to equation (1.1) is that there exists a nontrivial function $v$ with the following properties:

i) $v \in C\left([\alpha, \beta] ; R^{n}\right)$ and $v \in \mathbb{T}(\beta)$,

ii) $v^{\prime}$ exists and is continuous on $[\alpha, \beta]$ except for a finite number of discontinuities,

iii) $\int_{t}^{\beta} P(s) v(s) d s \geqslant v^{\prime}(t)$ with respect to the cone $K$ for $\alpha \leqslant t \leqslant \beta$.

As an application of Corollary 5.5, we present the following necessary condition for the absence of focal points. The proof is based on a technique of NEHARI [17].

Proposition 5.6. - Suppose $P(t)=\left(p_{i j}(t)\right), p_{i j}(t)>0,1 \leqslant i, j \leqslant n$, on $[\alpha, \beta] . A$ necessary condition for equation (1.1) to be disfocal on the interval $[\alpha, \beta)$ is that for $t \in[\alpha, \beta)$

$$
(i-\alpha) \int_{i}^{\beta}\|P(s)\|_{\infty} d s \leqslant 1
$$

Proof. - For fixed but arbitrary $x \in[\alpha, \beta)$, define

$$
w(s)= \begin{cases}\operatorname{col}(s-\alpha), & \alpha \leqslant s \leqslant x \leqslant \beta \\ \operatorname{col}(x-\alpha), & \alpha \leqslant x \leqslant s \leqslant \beta .\end{cases}
$$

For each $x \in[\alpha, \beta), w \in \mathfrak{S}(\beta)$ and

$$
w^{\prime}(s)= \begin{cases}1, & \alpha \leqslant s \leqslant x \leqslant \beta \\ 0, & \alpha \leqslant x \leqslant s \leqslant \beta .\end{cases}
$$

For $t \in[\alpha, \beta]$, define

$$
h(t)=w^{\prime}(t)-\int_{i}^{\beta} P(s) w(s) d s
$$


If $t \neq x$, then $h$ is differentiable and

$$
h^{\prime}(t)=P(t) w(t)>0
$$

Furthermore $h(x-)-h(x+)=1$. Since $h(\beta)=0, h(t) \leqslant 0$ for $x \leqslant t \leqslant \beta$, and, since $h^{\prime}(t)>0$ on $[\alpha, x), h(x) \leqslant 0$ would imply $h(t) \leqslant 0$ for $t \in[\alpha, x)$. It follows that if $h(x) \leqslant 0$, then $h(t) \leqslant 0$ for all $t \in[\alpha, \beta]$, i.e., for $t \in[\alpha, \beta]$

$$
w^{\prime}(t) \leqslant \int_{i}^{\beta} P(s) w(s) d s .
$$

Applying Corollary 5.5, $(\alpha, \beta]$ would contain a focal point for equation (1.1). Since the equation is assumed to be disfocal on $[\alpha, \beta)$, we must have $h(x) \geqslant 0$ or

$$
(x-\alpha) \int_{x}^{\beta} \sum_{j=1}^{n} p_{i j}(s) d s \leqslant 1, \quad i=1,2, \ldots, n .
$$

The inequality (5.10) is immediate, and the proof of the proposition is complete.

In view of Propositions 5.4 and 5.6, together with the equality (5.10), we have

$$
(t-\alpha) \int_{i}^{\beta}\|P(s)\|_{1} d s \leqslant 1
$$

is also a necessary condition for disfocality of equation (1.1) on $[\alpha, \beta)$. To obtain necessary conditions for equation (1.1) to be disfocal on the interval $[0, \infty)$, we note that (5.10) and (5.11) must hold for all $\beta>0$. In particular, we obtain

$$
t \int_{i}^{\infty}\|P(s)\|_{\infty} d s \leqslant 1, \quad t \int_{i}^{\infty}\|P(s)\|_{1} d s \leqslant 1 .
$$

For the one-dimensional case, the condition (5.12) reduces to the well-known sharp necessary condition of HILLE [9] for the scalar equation $y^{\prime \prime}+p_{0}(t) y=0, p_{0}(t) \geqslant 0$, $t \geqslant 0$, to be disconjugate, namely;

$$
t \int_{t}^{\infty} P_{0}(s) d s \leqslant 1 .
$$

\section{BIBLIOGRAPHY}

[1] S. AHMAD - A. LAZER, An N-dimensional extension of the separation and comparison theory to a class of nonselfadjoint systems, to appear.

[2] S. AHMAD - A. LAZER, On the components of extremal solutions of second order systems, SIAM Math. Anal., 8 (1977), pp. 16-23.

[3] S. Ahmad - C. Travis, Oscillation criteria for second order differential systems, Proc. Amer. Math. Loc., 71 (1978), pp. 247-252. 
[4] W. Athegretro - L. ERBe, Oscillation criteria for matrix differential inequalities, Canad. Math. Bull, 16 (1973), pp. 5-10.

[5] J. H. BARRETT, Systems-disconjugacy of a fourth-order differential equation, Proc. Amer. Math. Soc., 12 (1961), pp. 205-213.

[6] S-S. CHENG, Systems-conjugate and focal points of fourth order nonselfadjoint differential equations, Trans. Amer. Math. Soc., 223 (1976), pp. 155-165.

[7] R. GENTRY - C. TRAVIS, Existence and comparison of eigenvalues of $n$-th order linear differential equations, Bull. Amer. Math. Soc., 82, 2 (1976), pp. 350-352.

[8] R. GentrY - C. Travis, Comparison of eigenvalues associated with linear differential equations of arbitrary order, Trans. Amer. Math. Soc., 223 (1976), pp. 167-179.

[9] E. HmLE, Nonoscillation theorems, Trans. Amer. Math. Soc., 64 (1948), pp. 234-252.

[10] H. C. HOWARD, Some oscillation criteria for nonselfadjoint differential equations, to appear.

[11] M. KEENER, On the solutions of certain linear nonhomogeneous second-order differential equations, Appl. Anal., 1 (1971), pp. 57-63.

[12] M. KEENER - C. Travis, Focal points and positive cones for a class of $n$-th order differential equations, Trans. Amer. Math. Soc., 237 (1978), pp. 331-351.

[13] M. Kransnoselsiri, Positive solutions of operator equations, Fizmatgiz, Moseow, 1962; English transl., Noordhoff, Groningen, 1964.

[14] K. KReith, A nonselfadjoint dynamical system, Proc. Edinburgh Math. Soc., 19(1) (1974), pp. 77.87.

[15] K. KREITH, Oscillation properties of nonselfadjoint differential equations, Proc. Conf. on Differential Equations, Dundee, Scotland, Springer-Verlag Lecture Notes, no. 280 (1972), pp. 294-297.

[16] Z. NEeARI, Oscillation criteria for second order linear differential systems, Trans. Amer. Math. Soc., 85 (1957), pp. 428-445.

[17] Z. NeHaRI, Green's funetions and disconjugacy, Arch. Rat. Mech. Anal, 62 (1976), pp. 53-76.

[18] W. Rem, Oscillation eriteria for self-adjoint differential systems, Trans. Amer. Math. Soc., 101 (1961), pp. 91-106.

[19] J. SARABiA - E. ToRREs, On second order linear differential equations, J. Math. and Phys. Sciences, to appear.

[20] K. Schuitт - H. Smiтh, Positive solutions and conjugate points for systems of differential equations, Nonlin. Anal. Theor. Methods-Appl., 2 (1978), pp. 93-105.

[21] W. Simons, Disconjugacy criteria for systems of self-adjoint differential equations, J. London Math. Soc., (2), 6 (1973), pp. 373-381.

[22] C. Travis, Oomparison of eigenvalues for linear differential equations of order $2 n$, Trans. Amer. Math. Soc., 177 (1973), pp. 363-374. 